\title{
Comparison of the thoracic CT-based computational model with hyperpolarized Xenon-129 MRI and SPECT images to assess pulmonary ventilation in COPD patients
}

\author{
${ }^{1}$ Minsuok Kim, ${ }^{2,3}$ Ozkan Doganay, ${ }^{3}$ Tahreema Matin, ${ }^{1}$ Thomas Povey, ${ }^{2,3}$ Fergus Gleeson
}

1. Department of Engineering Science, University of Oxford, Oxford, UK

2. Department of Oncology, University of Oxford, Oxford, UK

3. Department of Radiology, The Churchill Hospital, Oxford University Hospitals NHS Trust, Oxford, UK

Introduction: Thoracic computed tomography (CT) is a clinically established diagnostic technique to detect structural pulmonary abnormalities but provides no functional pulmonary information.

Objectives: The current study aims to demonstrate the use of the CT-based full-scale airway network (FAN) flow model to assess ventilation in COPD patients and to compare the modelling results obtained with hyperpolarized Xenon-129 (HPX) magnetic resonance imaging (MRI) and singlephoton emission computed tomography (SPECT) imaging data.

Methods: Pulmonary ventilation in nine COPD patients was modelled using the CT-based FAN flow model. Pulmonary tissue density information extracted from the CT and Pulmonary Function Test (PFT) results were used for the patient-specific modelling. The ventilation calculated in the FAN model was compared to the PFT data, and the ventilation HPX-MRI and ventilation SPECT (V-SPECT) imaging.

Results: Pulmonary ventilation calculated from the FAN model was visually similar to ventilation demonstrated on the ventilation HPX-MRI and SPECT images (Fig 1), and statistically significant, Pearson correlation of the ventilation profiles between the FAN model and HPX-MRI and SPECT images, $\mathrm{r}_{\text {Mod-HPX }}=0.67 ; \mathrm{r}_{\text {Mod-SPE}}=0.61, \mathrm{P}<0.001$.

Conclusions: The CT-based FAN model utilizes structural imaging data to provide ventilation images comparable to functional imaging techniques. 

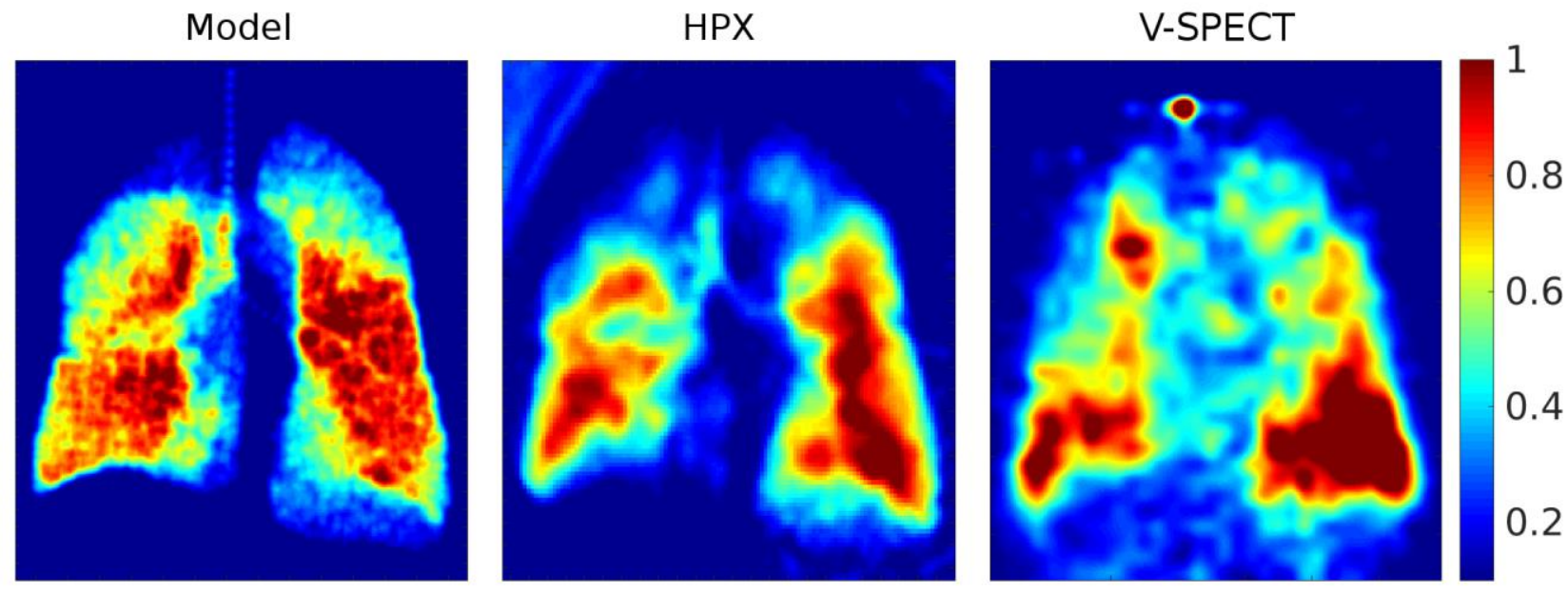

Figure 1. Comparison of the ventilation distribution projected on a coronal plane in a COPD patient's lung. 